\title{
Sequential modelling of ICRF wave near RF fields and asymptotic RF sheaths description for AUG ICRF antennas
}

\author{
Jonathan Jacquot ${ }^{1}$, Wouter Tierens ${ }^{1, \star}$, Wei Zhang ${ }^{1,2}$, Volodymyr Bobkov ${ }^{1}$, Laurent Colas ${ }^{3}$, Jean-Marie \\ Noterdaeme ${ }^{1,2}$, and The ASDEX Upgrade Team ${ }^{1}$ \\ ${ }^{1}$ Max-Planck-Institut für Plasmaphysik, Boltzmannstrasse 2, D-85748 Garching, Germany \\ ${ }^{2}$ Ghent University, Sint-Pietersnieuwstraat 41, Gent, Belgium \\ ${ }^{3}$ CEA, IRFM, F-13108 Saint Paul-lez-Durance, France
}

\begin{abstract}
A sequence of simulations is performed with RAPLICASOL and SSWICH to compare two AUG ICRF antennas. RAPLICASOL outputs have been used as input to SSWICH-SW for the AUG ICRF antennas. Using parallel electric field maps and the scattering matrix produced by RAPLICASOL, SSWICH-SW, reduced to its asymptotic part, is able to produce a $2 \mathrm{D}$ radial/poloidal map of the DC plasma potential accounting for the antenna input settings (total power, power balance, phasing). Two models of antennas are compared: 2-strap antenna vs 3-strap antenna. The 2D DC potential structures are correlated to structures of the parallel electric field map for different phasing and power balance. The overall DC plasma potential on the 3-strap antenna is lower due to better global RF currents compensation. Spatial proximity between regions of high RF electric field and regions where high DC plasma potentials are observed is an important factor for sheath rectification.
\end{abstract}

\section{Introduction}

Plasma heating with waves in the Ion Cyclotron Range of Frequencies (ICRF) is a convenient method to heat plasma and achieve the high temperatures required for a fusion reactor. However, in addition to the desired heating in the core plasma, spurious nonlinear interactions with the plasma edge and with plasma limiting boundaries have been observed in magnetic fusion devices equipped with ICRF antennas [1], such as JET, ASDEX Upgrade (AUG), Tore Supra, Alcator C-Mod. These interactions often determine the operational limits of the radio-frequency systems. Their most likely origin is RF-sheath rectification [2], which is suspected to produce enhanced sputtering (which causes impurity release [3-5]), increased heat fluxes [6] on the antennas as well as objects magnetically connected to the launcher, and Scrape Off Layer (SOL) density re-distribution [7] modifying the coupling properties of the antenna. Active strap RF currents and the induced image currents on the surrounding passive structures are a priori influencing the RF-sheaths' spatial topology and magnitude. For reliable ICRF operation at high power over long pulses in metallic environment, the physical mechanisms and their interplay that underlie these spurious interactions must be understood and minimized. The present paper presents simulations results

^e-mail: wouter.tierens@ipp.mpg.de for two ASDEX Upgrade antennas (so called 2-strap and 3-straps antenna, see figure 1) obtained with two codes:

- RAPLICASOL (Radiofrequency wAve couPLing for Ion Cyclotron Antenna in Scrape-Off-Layer) to solve Maxwell's equations in the cold plasma approximation without sheaths or any non-linear phenomenon.

- SSWICH (Self-consistent Sheaths and Waves for Ion Cyclotron Heating) to self-consistently determine the non linear response of the interaction of $\mathrm{RF}$ waves with the edge plasma (SOL).

\section{RAPLICASOL}

RAPLICASOL [8] is a 3D finite-element model implemented in COMSOL[9], which solves Maxwell's equations in the cold plasma approximation in the neighbourhood of a realistic 3D antenna geometry as shown in figure 1. Being restricted to cold plasma, RAPLICASOL cannot model the core plasma. Instead an absorbing boundary layer is used at the core plasma (a Perfectly Matched Layer [10] or PML). On all other boundaries, metallic boundary conditions are enforced. Waves are first excited from port 1, and then from port 2 , which gives us all information required to determine the scattering parameters $(S$ matrix) and related quantities. To further decrease 

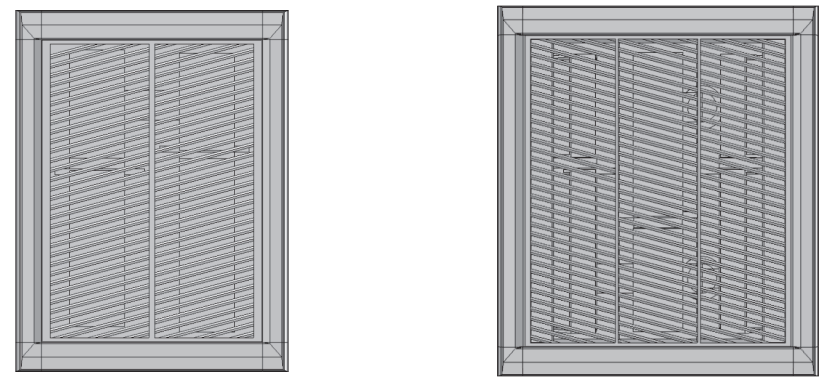

Figure 1. 3D model of the 2-strap antenna (left) and the 3 -strap antenna (right). The 2 side straps of the 3-strap antennas are fed from the same port.

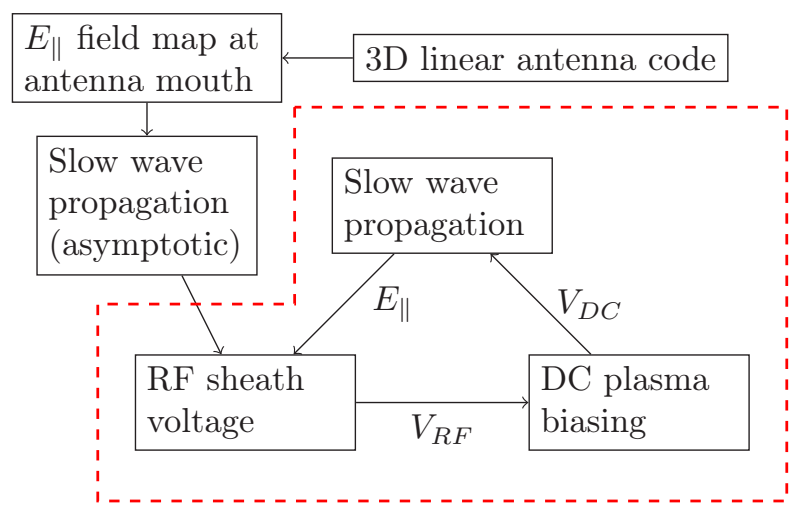

Figure 2. Schematic representation of the algorithm used in the SSWICH code. The three fluids are solved in their own module and are coupled together through RF sheath properties. An asymptotic version of the SW module initializes the loop to ensure convergence.

the memory requirements, an iterative solver is used [11].

A fairly detailled 3D model of the antenna geometry, as shown in figure 1 , is imported into the model and placed in vacuum. To avoid numerical resolution issues in the private region of the antenna and in particular near the LH resonance [12], the density is assumed to be 0 up to the leading edge of the antenna.

\section{SSWICH-SW asymptotic}

The SSWICH [13-15] code follows a three-field approach to self-consistently model the interplay between RF waves and edge plasma DC biasing. In the SSWICH-SW version which is used in this study, the $\mathrm{RF}$ waves are reduced to the slow magnetosonic wave (SW) only. The simulation domain is a set of straight open magnetic field lines in the SOL of magnetized plasma in the vicinity of an IC antenna. This domain contains protruding material objects able to intersect magnetic field lines. The inclusion of the limiters allows to distinguish long field lines passing in front of the antennas from short field lines connected to the limiters or in between the limiters. The magnetic field lines are either perpendicular or parallel to the boundaries in order to be consistent with the assumption of the slow wave only[16]. Inside this domain and as shown on figure 2, three fields are solved for: the RF parallel electric field $E_{\|}$, an oscillating sheath voltage $V_{R F}$ (both varying as as $\exp \left(-i \omega_{0} t\right)$ at the wave pulsation $\left.\omega_{0}\right)$ and the DC plasma potential $V_{D C}$. Sheaths at both ends of open flux tubes are described by RF and DC sheaths boundary conditions [17, 18]. The non-linear I-V characteristic of the sheaths couples the RF and DC quantities. More details can be found in [13]. The excitation field map is computed in the present paper with the antenna code RAPLICASOL. The physical model of SSWICH-SW is implemented using the COMSOL Finite Element solver. In the present study, only the asymptotic part of SSWICH is considered in order to reduce the simulation time of an extensive number of simulations (the loop in figure 2 only runs once). Due to limited computational resources, an automated multi-2D approach was used, whereby several radial/parallel planes at consecutive poloidal positions are solved independently, assuming the poloidal derivatives can be neglected with respect to radial gradient in all the equations.

\section{Simulations results interfacing SSWICH-SW with RAPLICASOL}

Numerical simulations were performed to compare both models of AUG antennas at $30 \mathrm{MHz}$ and $B=$ $1.917 \mathrm{~T}$. This frequency is the standard operating frequency for $\mathrm{H}$ minority. The imported density profile corresponds to the scenario of \#31278. Although SSWICH and RAPLICASOL can handle 3D density profiles [19], only a 1D radial density profile is considered in this paper. All calculations are scaled to $1 \mathrm{MW}$ coupled power to the plasma. Results from RAPLICASOL simulations are shown in figure 3 displaying the RF parallel electric field $E_{\|}$in front of the Faraday screen in between the antenna limiters. The RF electric field pattern outside the limiters is neglected. The 2-strap antenna is configured in a standard dipole phasing (i.e. $0^{\circ}$ phase difference between both ports) and a balanced power distribution, whereas the three straps antenna is configured with twice more power on the central strap compared to the side straps in dipole phasing. In both cases, the fieldmap is a linear superposition of the simulated fieldmap for each port one at a time normalized using the scattering matrix. It clearly shows that the $E_{\|}$field, generated by induced RF currents, near the limiters is mitigated for the 3-strap antenna. The presence of the Faraday screen bars is creating a small scale field structure in the capacitive E fields generated in the strap boxes. The modulation of this pattern is following the number of bars in the geometry. These fieldmaps were then the source files for SSWICH simulations. All parameters are identical for both antennas. The vertical step size between two SSWICH simulations is fixed to $1 \mathrm{~cm}$, which is approximately to the size of a Faraday screen bar, and is the smallest length scale on which we should expect $V_{D C}$ variations. 

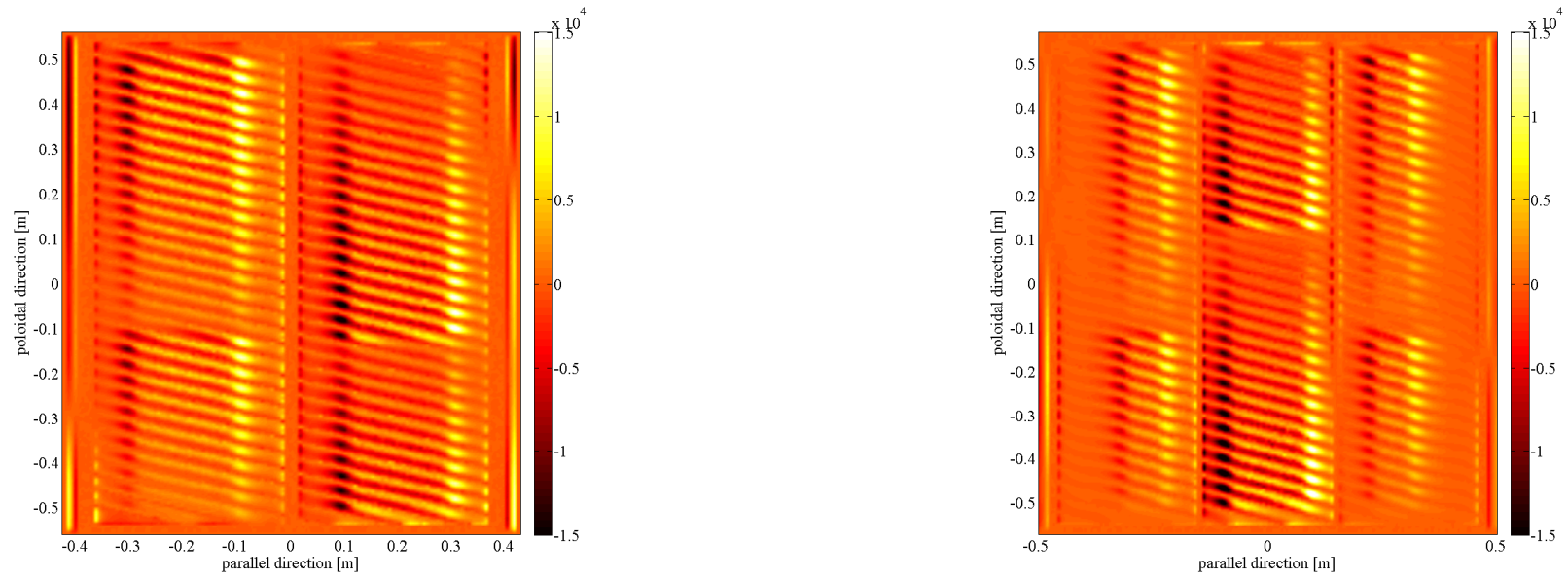

Figure 3. 2D map (poloidal/toroidal) in front of the Faraday screen of the real part of the parallel RF electric field for the 2-strap antenna (left) and 3-strap antenna (right). Coupled power $=1 \mathrm{MW}$, dipole phasing, power balance 0.5:0.5 (left) and 2/3:1/3 (right).
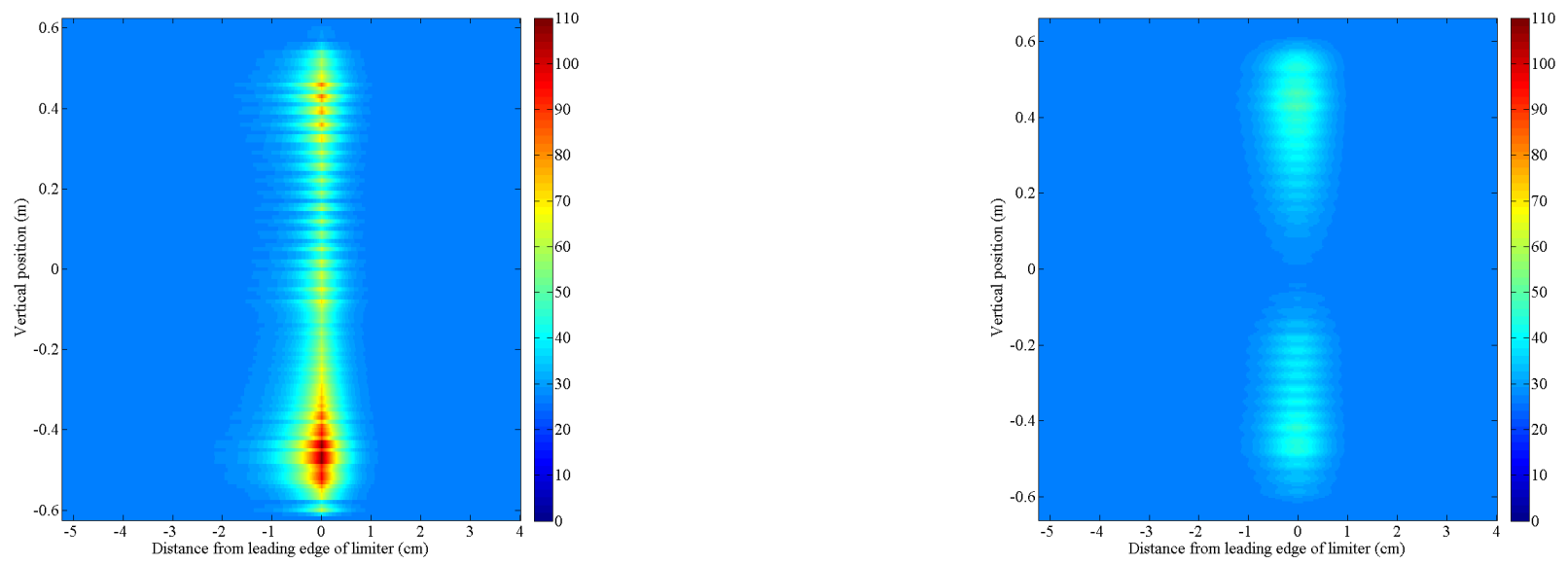

Figure 4. 2D map (poloidal/radial) of the DC plasma potential on the outer side of the right limiter simulated by the sequence RAPLICASOL/SSWICH-SW computed with the fieldmaps in figure 3. The left figure is for the 2 -strap antenna while the figure for the 3-strap antenna is on the right. Positive distance from the leading edge denotes the direction towards the center of the torus.

The finite DC conductivity tensor establishes a circulation of DC currents from the private region of the antenna to the rest of the SOL. The compensation of RF induced currents on the 3-Strap antenna indeed translates into a significant decrease of the DC plasma potential $V_{D C}$ in SSWICH-SW on the outer side of the antenna limiter, as seen on figure $4(60 \mathrm{~V}$ compared to $110 \mathrm{~V})$. The absolute value of $V_{D C}$ is severely affected by the local density [19], but also by poorly constrained parameters in SSWICH [13]. More interesting is the relative comparison. A doublehump structure can be seen with maxima near the top and bottom of the antenna for both antennas where the highest parallel electric field are present. It results that toroidal and poloidal proximity to regions of high $E_{\|}$is indeed dominant [20]. The topology of this structure has been observed on different ICRF antennas $[13,20]$.

Both figures contain vertically small scale structures that are attributed to the Faraday screen bars. These modulations of $V_{D C}$ can occur due to electric fields further toroidally from the limiters away and follow the modulation in the electric field map. The relative visibility of this modulation is also related to the multi-2D procedure in SSWICH. In the case of the 3D version of SSWICH, poloidal transport properties (i.e. DC transverse conductivity that is not only radial but also poloidal) would likely smooth the modulation.

A scan of the maximum value of $V_{D C}$ vs. the phasing and power balance leads to figure 5 . The power balance, contrary to the convention in [4], is here defined as $p_{b}=\frac{P_{\text {outer }}}{P_{\text {outer }}+P_{\text {center }}}$ for the 3-strap antenna. 

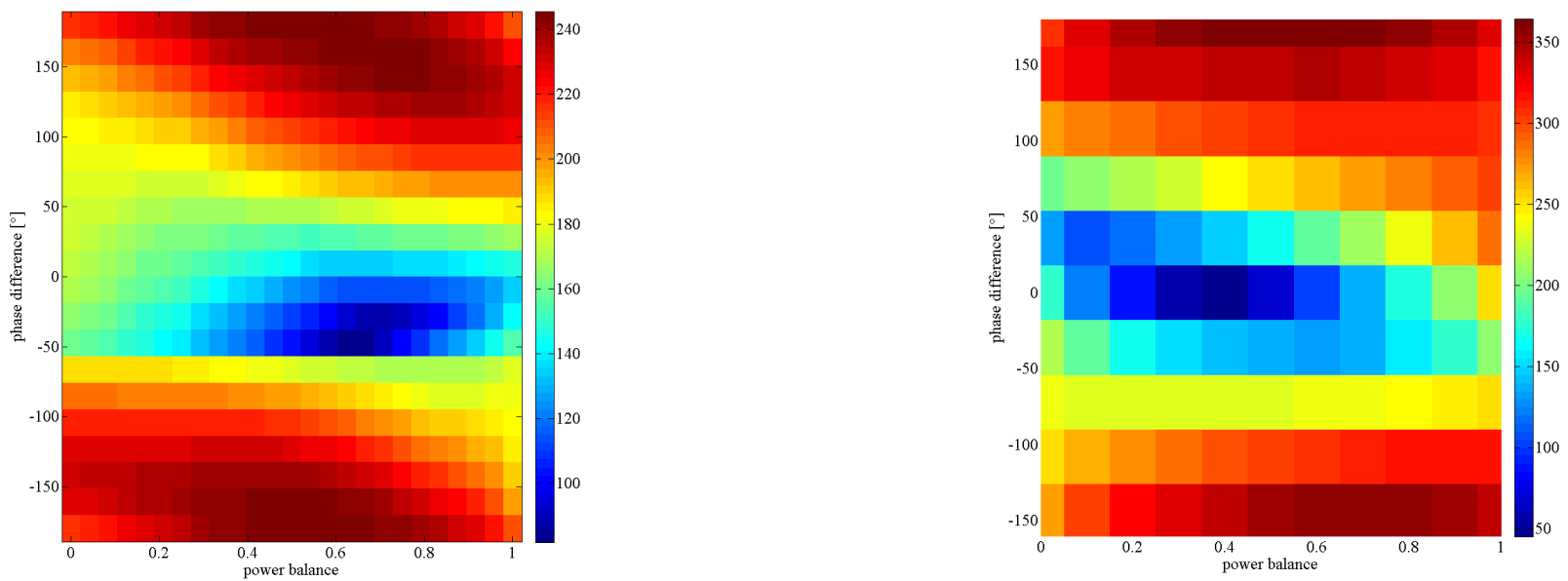

Figure 5. Maximum DC plasma potential $V_{D C}$ of the outer side of the right limiter vs. power balance and phasing for the 2-strap antenna (left) and the 3-strap antenna (right). Dipole phasing corresponds to a zero phase difference.

A similar definition is used for the 2-strap antenna: $p_{b}=\frac{P_{\text {right }}}{P_{\text {right }}+P_{\text {left }}}$. For the 3 -strap antenna, the maximal $V_{D C}$ is minimized close to dipole phasing and $p_{b}=\frac{1}{3}$. It does also corresponds to the minimum for $E_{\|}$as well as experimental values [4]. The situation seems to get worse than for the 2-strap antenna in case of bad power balance and phasing settings.

\section{Conclusions and discussion}

Simulations were performed with RAPLICASOL and SSWICH-SW to characterize two AUG ICRF antennas. Qualitative features of the experimental observation are well captured by the calculations. Progress was also made for quantitative predictions. We believe the accuracy is constrained by uncertainties in the experimental input and loosely constrained numerical parameters in SSWICH. Repeating the scan in power balance and phasing with a $3 \mathrm{D}$ density [19] could help to close the discrepancy. SSWICH-SW only accounts for the slow wave and its mechanism for generating transverse DC current is likely oversimplified. Furthermore, the RF electric field outside the antenna limiters are presently neglected. Replacing it with SSWICH-FW (Full Wave) [16] would bring the fast wave into the mix and open the way to a $3 \mathrm{D}$ SSWICH-FW.

Acknowledgments This work has been carried out within the framework of the EUROfusion Consortium and has received funding from the European research and training programme under grant agreement $\mathrm{N}^{\circ} 633053$. The views and opinions expressed herein do not necessarily reflect those of the European Commission.

\section{References}

[1] J.M. Noterdaeme, G.V. Oost, Plasma Physics and Controlled Fusion 35, 1481 (1993)
[2] F. Perkins, Nuclear Fusion 29, 583 (1989)

[3] D'Ippolito et al., Plasma Physics and Controlled Fusion 33, 607 (1991)

[4] V. Bobkov et al., Plasma Physics and Controlled Fusion 59, 014022 (2017)

[5] V. Bobkov et al., Nuclear Fusion 53, 093018 (2013)

[6] L. Colas et al., Fusion Science and Technology 56, 1173 (2009)

[7] W. Zhang et al., Nuclear Fusion 57, 116048 (2017)

[8] J. Jacquot et al., Proc. 21th RF Top. Conf 1689 (2015)

[9] https://www.comsol.com/

[10] J. Berenger, Journal of computational physics 114, 185 (1994)

[11] Y. Saad, Iterative methods for sparse linear systems (SIAM, 2003)

[12] L. Lu et al., Plasma Physics and Controlled Fusion 58, 055001 (2016)

[13] J. Jacquot et al., Physics of Plasmas 21, 061509 (2014)

[14] L. Colas et al., Physics of Plasmas 19, 092505 (2012)

[15] J. Jacquot, Ph.D. thesis, Université de Lorraine (2013)

[16] L. Lu, ECA (submitted to Plasma Physics and Controlled Fusion) 40A, 2.068 (2016)

[17] D. D'Ippolito, J. Myra, Physics of plasmas 13, 102508 (2006)

[18] M.A. Lieberman, IEEE Transactions on Plasma Science 16, 638 (1988)

[19] W. Tierens et al., This conference (2017)

[20] L. Colas et al., Plasma Physics and Controlled Fusion 59, 025014 (2017) 